\title{
Magnetic Properties of (Zn,Mn)Te Semimagnetic Alloy Co-Doped with Chromium
}

\author{
A. Avdonin, Le Van Khoi, W. Knoff and R.R. GaŁązKa \\ Institute of Physics, Polish Academy of Sciences, al. Lotników 32/46, 02-668 Warsaw, Poland
}

\begin{abstract}
Results of simultaneous doping of ZnTe with manganese and chromium are presented. An increase of magnetization of ferromagnetic Cr-related clusters with manganese concentration is observed. Phosphorus doping prevents the formation of ferromagnetic clusters.
\end{abstract}

PACS numbers: 78.55.Et, 75.50.Pp, 75.60.Ej

\section{Introduction}

The results of magnetic and magnetoreflectance measurements of ZnTe simultaneously doped with manganese and chromium are presented. A similar approach of simultaneous doping of CdTe with manganese and chromium was recently reported in [1].

\section{Experimental}

The crystals were grown by Bridgman method from a mixture of $\mathrm{ZnTe}, \mathrm{MnTe}, \mathrm{Cr}$ and Te. Some of the samples were additionally doped with phosphorus in order to change the free carrier concentration. For all samples nominal content in the charge was $\mathrm{Mn}-0.05, \mathrm{Cr}-$ 0.02 . Concentration of phosphorus was varied between 0 and $10^{20} \mathrm{~cm}^{-3}$. As a reference $(\mathrm{ZnMn}) \mathrm{Te}$ and $(\mathrm{ZnCr}) \mathrm{Te}$ samples were used, also grown by the Bridgman method. For $(\mathrm{ZnMn}) \mathrm{Te}$ the charge consisted of $\mathrm{ZnTe}$ and $\mathrm{MnTe}$, and for $(\mathrm{ZnCr}) \mathrm{Te}$ the charge consisted of $\mathrm{ZnTe}, \mathrm{Cr}$ and Te. In the (ZnMn)Te sample the manganese content was 0.05 , and in $(\mathrm{ZnCr}) \mathrm{Te}$ sample the chromium content was 0.02 . The reference $(\mathrm{ZnMn}) \mathrm{Te}$ and $(\mathrm{ZnCr}) \mathrm{Te}$ samples were not doped with phosphorus. Although Mn is fully soluble in $\mathrm{ZnTe}$, the solubility of $\mathrm{Cr}$ in $\mathrm{ZnTe}$ is limited to about 0.005 [2]. Thus the real chromium concentration in the samples is less than its concentration in the charge.

\section{Results and discussion}

The magnetization measurements were done on a SQUID magnetometer. For comparison the same measurements were performed on $(\mathrm{ZnCr}) \mathrm{Te}$ samples. As it was previously reported (e.g. $[3,4])$, chromium doped ZnTe samples possess ferromagnetic properties even at room temperature. However, such ferromagnetism is commonly attributed to CrTe precipitations or chromium rich clusters of zinc-blende structure. The samples additionally doped with manganese have similar properties. A comparison of hysteresis curves of $(\mathrm{ZnCr}) \mathrm{Te}$

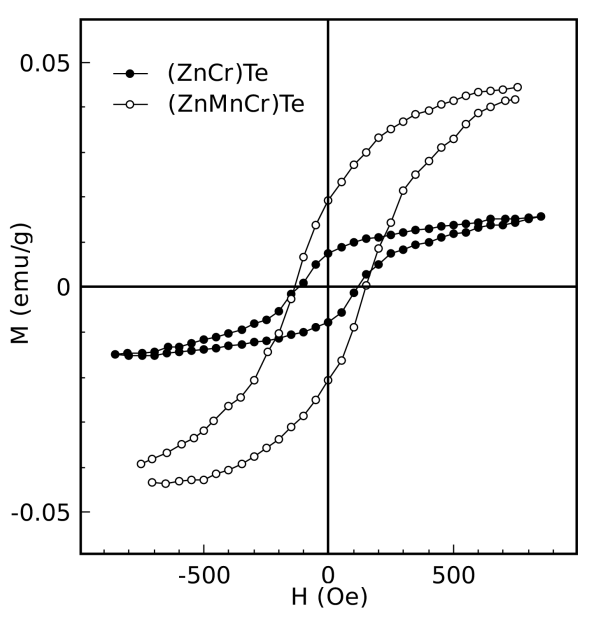

Fig. 1. Hysteresis curves measured at $150 \mathrm{~K}$. Filled circles - $(\mathrm{ZnCr}) \mathrm{Te}$, open circles - $\mathrm{ZnMnCr}) \mathrm{Te}$.

and $(\mathrm{ZnMnCr}) \mathrm{Te}$ is presented in Fig. 1. An interesting observation is that the saturation magnetization in $(\mathrm{ZnMnCr}) \mathrm{Te}$ is three times stronger than in $(\mathrm{ZnCr}) \mathrm{Te}$. There might be two possible explanations: (i) doping with manganese increases the solubility of chromium or (ii) in the presence of chromium, manganese takes part in formation of ferromagnetic clusters. In view of magnetoreflectance result (presented below) the latter explanation is more probable.

In samples additionally doped with phosphorus the ferromagnetic signal decreases when concentration of phosphorus is $10^{18} \mathrm{~cm}^{-3}$ and completely disappears when it is greater than $10^{19} \mathrm{~cm}^{-3}$ (see Fig. 2). In the beginning of this process when concentration of phosphorus is not very large $\left(10^{18} \mathrm{~cm}^{-3}\right)$ one can see on the hysteresis curve just weakening of the coercive field and the unchanged saturation magnetization. This means that transition from ferromagnetic to non-ferromagnetic passes through the 


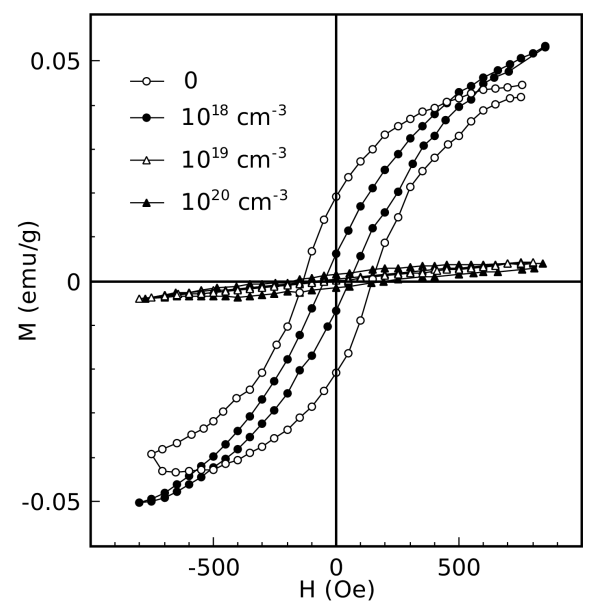

Fig. 2. Hysteresis curves measured at $150 \mathrm{~K}$ on samples with different phosphorus concentration.

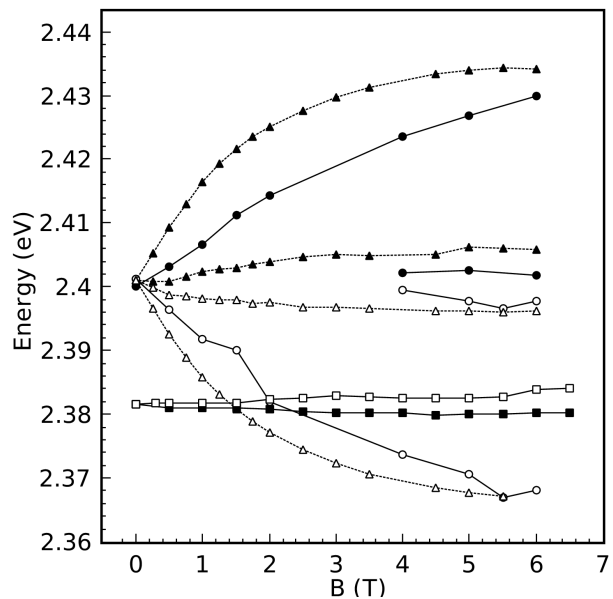

Fig. 3. Free exciton energy as a function of magnetic field at $5 \mathrm{~K}$. Circles - $(\mathrm{ZnMnCr}) \mathrm{Te}$, squares $(\mathrm{ZnCr}) \mathrm{Te}$, triangles - ( $\mathrm{ZnMn}) \mathrm{Te}$. Filled symbols $\sigma^{-}$light polarization, open symbols $-\sigma^{+}$light polarization.

formation of smaller super-paramagnetic clusters. Disappearance of ferromagnetism in $p$-doped samples is in accordance with the theory reported in [4], where it is demonstrated that formation of ferromagnetic clusters in $(\mathrm{ZnCr}) \mathrm{Te}$ is possible only in undoped or $n$-doped samples and is due to the process of so-called "spinodal decomposition".

Magnetoreflectance measurements were performed only on undoped ( $\mathrm{ZnMnCr}$ )Te sample because in heavily doped samples the free excitons are screened by free carriers. The excitonic magnetoreflectance gives an opportunity to measure the constants of $p-d$ exchange interaction between magnetic ions and holes $(\beta)$. It is known that in $(\mathrm{ZnMn}) \mathrm{Te}$ and $(\mathrm{ZnCr}) \mathrm{Te}$ those constants have opposite sign and it was tempting to see what happens with sample simultaneously doped with Mn and Cr. The result of the measurement is presented in Fig. 3 along with two other measurements of ( $\mathrm{ZnMn}) \mathrm{Te}$ and ( $\mathrm{ZnCr}) \mathrm{Te}$ for comparison. One can see that the Zeeman splitting of $(\mathrm{ZnCr}) \mathrm{Te}$ is opposite to that of $(\mathrm{ZnMn}) \mathrm{Te}$ and $(\mathrm{ZnMnCr}) \mathrm{Te}$. Samples (ZnMn)Te and (ZnMnCr)Te have the same Mn concentration (they have the same zero-field exciton energy). However, the splitting of the latter is smaller, especially at low fields. To explain that, it should be mentioned that there is a difference of magnetization measured in field cooling and zero field cooling conditions. Thus we assume that a part of Mn magnetic moments is frozen at $5 \mathrm{~K}$ and get aligned only at higher magnetic fields. That would explain the flat slope of the $(\mathrm{ZnMnCr}) \mathrm{Te}$ in comparison with $(\mathrm{ZnMn}) \mathrm{Te}$, and would confirm that $\mathrm{Mn}$ along with $\mathrm{Cr}$ takes part in formation of ferromagnetic clusters.

\section{References}

[1] S. Shen, X. Liu, Y.J. Cho, J.K. Furdyna, M. Dobrowolska, Y.H. Hwang, Y.H. Um, Appl. Phys. Lett. 94, 142507 (2009).

[2] G. Krishnaiah, N. Madhusudhana Rao, D. Raja Reddy, B.K. Reddy, P. Sreedhara Reddy, J. Cryst. Growth 310, 26 (2008).

[3] H. Saito, V. Zayets, S. Yamagata, K. Ando, Phys. Rev. Lett. 90, 207202 (2003).

[4] S. Kuroda, N. Nishizawa, K. Takita, M. Mitome, Y. Bando, K. Osuch, T. Dietl, Nature Mater. 6, 440 (2007). 